\title{
An inequality involving a ratio of zeta functions
}

\author{
József Sándor \\ Department of Mathematics, Babeş-Bolyai University \\ Str. Kogălniceanu nr.1, 400084 Cluj, Romania \\ email: jsandor@math.ubbcluj.ro
}

Received: 7 October 2017

Accepted: 30 August 2018

Abstract: We prove an inequality for a ratio of zeta functions. This extends a classical result (see [2]). The method is based on Dirichlet series, combined with real analysis.

Keywords: Riemann zeta function, Dirichlet series, Inequalities for real functions.

2010 Mathematics Subject Classification: 11A25, 11N37, 26D20.

Let $\omega(n)$ denote the number of distinct prime divisors of $n$. Then $\omega(1)=0$ and $\omega(n)$ is an additive function, i.e.

$$
\omega(m n)=\omega(m)+\omega(n) \text { for all }(m, n)=1 .
$$

This implies immediately that the function

$$
f(n)=k^{\omega(n)}
$$

(where $k \geq 2$ is fixed) is a multiplicative function, i.e. satisfies the functional equation

$$
f(m n)=f(m) \cdot f(n) \text { for all }(m, n)=1,
$$

where $f(1)=1$.

A general Dirichlet series is an infinite series of type $\sum_{n=1}^{\infty} \frac{a_{n}}{n^{s}}$, where $s \in \mathbb{C}$ is such that the series is convergent. For $a_{n}=1$, we get the Riemann zeta function

$$
\zeta(s)=\sum_{n=1}^{\infty} \frac{1}{n^{s}}
$$


which is convergent e.g. for all $\operatorname{Re} s>1$. Another Dirichlet series is obtained when

$$
a_{n}=f(n)=k^{\omega(n)} .
$$

We will prove in what follows the following result:

Theorem. Let $s>1$ a fixed positive integer. Then one has the inequality

$$
\sum_{k=1}^{\infty} \frac{k^{\omega(n)}}{n^{s}} \leq \frac{\zeta^{k}(s)}{\zeta(k s)}
$$

with equality only for $k=2$.

For the proof, the following well-known result will be applied (see e.g. [1]).

Lemma 1. Let $f$ be a multiplicative arithmetical function, and let the series $\sum_{n=1}^{\infty} f(n)$ be absolutely convergent. Then we have the identity:

$$
\sum_{n=1}^{\infty} f(n)=\prod_{p \text { prime }}\left(1+f(p)+f\left(p^{2}\right)+\ldots\right) .
$$

We shall need also the following auxiliary result:

Lemma 2. Let $0<x \leq \frac{1}{2}$ and $k \geq 2$. Then

$$
1-x^{k} \geq(1-x)^{k-1}[1+x(k-1)] .
$$

Inequality (4) may be written also as

$$
1-x^{k} \geq(1-x)^{k-1}[(1-x+k x)]=(1-x)^{k}+k x(1-x)^{k-1} .
$$

Let us define

$$
g(x)=x^{k}=(1-x)^{k}+k x(1-x)^{k-1}, g:[0,1] \rightarrow \mathbb{R} .
$$

We have to prove that $g(x) \leq 1$. One has

$$
g(1)=g(0)=1 \quad \text { and } \quad g^{\prime}(x)=k x\left[x^{k-2}-(k-1)(1-x)^{k-2}\right] .
$$

Remark that, as $0<x \leq \frac{1}{2}$, we have $0<x \leq 1-x$, so

$$
x^{k-2} \leq(1-x)^{k-2} \leq(k-1)(1-x)^{k-2},
$$

with equality only for $k=2$. Thus we get $g^{\prime}(x) \leq 0$, implying

$$
g(x) \leq g(0)=1 \text {. }
$$

Remark. The above proof shows that there is equality in (4) only for $k=2$.

Proof of Theorem. Letting

$$
f(n)=\frac{k^{\omega(n)}}{n^{s}}
$$

in Lemma 1, we get 


$$
\sum_{n=1}^{\infty} \frac{k^{\omega(n)}}{n^{s}}=\prod_{p \text { prime }}\left(1+\frac{k}{p^{s}}+\frac{k}{p^{2 s}}+\ldots\right)
$$

For $f(n)=\frac{1}{n^{s}}$ in the same Lemma 1, we get Euler's identity

$$
\sum_{k=1}^{\infty} \frac{1}{n^{s}}=\prod_{p \text { prime }}\left(1+\frac{k}{p^{s}}+\frac{k}{p^{2 s}}+\ldots\right)=\prod_{p \text { prime }} \frac{1}{1-\frac{1}{p^{s}}} .
$$

Thus, by using Euler's identity, we get

$$
\zeta(k s)=\prod_{p \text { prime }} \frac{1}{1-\frac{1}{p^{k s}}},
$$

i.e.,

$$
\frac{\zeta^{k}(s)}{\zeta(k s)}=\prod_{p \text { prime }} \frac{1-\frac{1}{p^{k s}}}{\left(1-\frac{1}{p^{s}}\right)^{k}}
$$

Put now $x=\frac{1}{p^{s}}$. As $s>1$ and $p \geq 2$, clearly $x<\frac{1}{2}$. So we can apply Lemma 2 , which implies

$$
\frac{1-x^{k}}{(1-x)^{k}} \geq \frac{1+x(k-1)}{1-x}
$$

In relation (5) one has

$$
\begin{aligned}
1+\frac{k}{p^{s}}+\frac{k}{p^{2 s}}+\ldots & =1+k x+k x^{2}+\ldots \\
& =1+k x\left(1+x+x^{2}+\ldots\right) \\
& =1+\frac{k x}{x-1}=\frac{1+x(k-1)}{x-1} .
\end{aligned}
$$

By relations (6) and (7), this implies inequality (2), finishing the proof of Theorem.

Remark. For $k=2$ we get the known identity (see [2])

$$
\sum_{k=1}^{\infty} \frac{2^{\omega(n)}}{n^{s}}=\frac{\zeta^{2}(s)}{\zeta(2 s)}
$$

\section{References}

[1] Hardy, G. H., \& Wright, E. M. (1964) An Introduction to the Theory of Numbers, Oxford Univ. Press.

[2] Titchmarsh, E. C. (1951) The Theory of the Riemann Zeta Function, Oxford. 